\title{
GROUPS GENERATED BY TWO OPERATORS OF THE SAME PRIME ORDER SUCH THAT THE CONJUGATES OF ONE UNDER THE POWERS OF THE OTHER ARE COMMUTATIVE.
}

BY PROFESSOR G. A. MILLER.

(Read before the American Mathematical Society, December 28, 1916.)

\section{$\S 1$. Introduction.}

IN a previous article,* devoted to a study of conjugate operators, attention was called to the fact that a complete set of conjugate operators which are not all relatively commutative is transformed under the group according to a substitution group which is at most triply transitive. It is easy to prove that this substitution group is at most doubly transitive, and, as the $p$ conjugates of order 2 under the holomorph of the group of the prime order $p$ are transformed under this holomorph according to a doubly transitive group, the substitution group in question can actually be doubly transitive.

The most general definition of a complete set of conjugate operators, or conjugate subgroups, of a group $G$ is that the set is composed of the totality of operators, or subgroups, which correspond under one or more of the possible automorphisms of $G$. It is, however, usually assumed that such a set is composed of the totality of operators, or subgroups, which correspond under at least one of the inner automorphisms of $G$. Since every possible automorphism of any group is an inner automorphism under its holomorph, this difference in the possible definitions of the expression complete set of conjugates does not affect the general theorems relating to such sets.

The theorem mentioned in the first paragraph can be materially extended by the consideration of a complete set of conjugate operators involving an operator which is commutative with at least one of the others without being commutative with all of them. When this condition is satisfied the group of transformations of the set is always simply transitive. To prove this let $s_{1}, s_{2}$ represent two commutative operators of

* Jahresbericht der Deutschen Mathematiker-Vereinigung, vol. 19 (1910), p. 318. 
the set of conjugates under consideration, while $s_{2}, s_{3}$ are two non-commutative operators of this set; then the group $G$ containing these conjugates cannot involve any operator $t$ which transforms $s_{1}$ into $s_{2}$ and also $s_{2}$ into $s_{3}$, since the equation

implies

$$
s_{1} s_{2}=s_{2} s_{1}
$$

$$
t^{-1} s_{1} s_{2} t=t^{-1} s_{2} s_{1} t, \quad \text { or } \quad t^{-1} s_{1} t \cdot t^{-1} s_{2} t=t^{-1} s_{2} t \cdot t^{-1} s_{1} t .
$$

Hence the following theorem has been proved: If a complete set of conjugate operators under any group $G$ is transformed according to a multiply transitive substitution group by the operators of $G$ then either every possible pair of these conjugates is composed of commutative operators or no one of these conjugates is commutative with any operator of the set besides itself.

\section{§2. Conjugate Sets Having Special Properties.}

When one operator of a set of conjugate operators under a group $G$ is commutative with each of the others except one, or with only one of the others, then it is clearly possible to arrange all the operators of the set in distinct pairs which constitute systems of imprimitivity of the substitution group according to which the operators of the set are transformed under $G$. In particular, it results from this that if a complete set of conjugate operators under a group is composed of an odd number of operators it is not possible that one operator be commutative with each of the others save one, or that one of these operators be commutative with one and only one other operator of the set.

If a complete set of conjugate operators under $G$ contains two and only two operators which are not commutative with some one of these conjugates then these two operators must be commutative with each other unless the set of conjugates is transformed under $G$ according to an imprimitive group having three elements in a system of imprimitivity. In particular, if the number of operators in a complete set of conjugates under $G$ is not divisible by 3 and if this set involves two and only two operators which are not commutative with a certain other operator of the set, then these two operators must be commutative with each other. As a more special case we may note the following theorem: If exactly $p-2, p$ being a 
prime number $>3$, of the operators in a complete set of $p$ conjugate operators are commutative with some one of these conjugates the remaining two operators of the set must be commutative with each other.

With slight modifications the arguments of the preceding paragraph apply to the case when in a complete set of conjugate operators under a group one of these conjugates is commutative with two and only two of the others. If these two operators are commutative with each other, the total set of conjugates is clearly transformed according to an imprimitive substitution group, having three elements in a system, by all the operators of the group in question. Hence it results that in a set of $p$ conjugates under the powers of an operator of the prime order $p$ it is not possible that one of these conjugates is commutative with two and only two of the others if these two are commutative and $p>3$. In particular if a set of 5 conjugates under the powers of an operator of order 5 includes an operator which is commutative with at least one of the others without being commutative with each of them, then this operator is commutative with exactly two other operators and these two operators are non-commutative while the remaining two operators must be commutative.

If $t$ transforms $s$ into an operator which is commutative with $s$, then $t^{-1}$ must evidently also transform $s$ into an operator which is commutative with $s$. Hence a complete set of conjugate operators under the powers of an operator of odd order must always involve an odd number of operators which are commutative with a particular operator of the set. The preceding theorems relating to a complete set of conjugate operators clearly apply also to a complete set of conjugate subgroups if we replace the term commutative by the term permutable.

\section{§3. Determination of the Possible Groups Generated by Two Operators of the Same Prime Order such that the Conjugates of One under the Other are Commutative.}

Let $s_{1}$ and $s_{2}$ be two distinct operators of the same prime order $p$, and suppose that the $p$ conjugates of $s_{2}$ under the powers of $s_{1}$ are commutative with each other. There are two possible cases to be considered. In the first case these $p$ conjugates of $s_{2}$ are identical and the group generated by $s_{1}$ and $s_{2}$ is either the group of order $p$ generated by $s_{2}$ or the 
non-cyclic group of order $p^{2}$, as $s_{1}$ generates or does not generate $s_{2}$. This case is practically trivial and requires no further consideration.

In the second possible case the $p$ conjugates of $s_{2}$ under the powers of $s_{1}$ are distinct. In this case the order of the group generated by $s_{1}$ and $s_{2}$ cannot be less than $p^{3}$ nor greater than $p^{p+1}$, since the $p$ conjugates in question cannot generate a group whose order is less than $p^{2}$ or greater than $p^{p}$. When $p=2$, the upper and lower limits for the orders of the possible groups are equal, and hence the octic group is the only possible group in this case. In general, for every prime number $p$ there are $p-1$ groups which are generated by two non-commutative operators of order $p$ satisfying the condition that the $p$ conjugates of one of these operators under the powers of the other are commutative.

Since the conjugates of $s_{2}$ under the powers of $s_{1}$ are commutative, it results that the powers of $s_{2} s_{1}$ may be represented as follows, if $s_{1} s_{2} s_{1}^{-1}=t_{1} s_{2}$, and $s_{1} t_{a} s_{1}^{-1}=t_{a+1} t_{a}$ :

$$
\begin{gathered}
s_{2} s_{1}=s_{2} s_{1}, \quad\left(s_{2} s_{1}\right)^{2}=s_{2} s_{1} s_{2} s_{1}{ }^{-1} s_{1}{ }^{2}=t_{1} s_{2}{ }^{2} s_{1}{ }^{2}, \quad\left(s_{2} s_{1}\right)^{3}=t_{2} t_{1}{ }^{3} s_{2}{ }^{3} s_{1}{ }^{3}, \\
\left.\left(s_{2} s_{1}\right)^{n}=t_{n-1} t_{n-2}^{n} \cdots t_{n-r}^{(n-1) \ldots(n-r) !}\right) \\
\cdots s_{2}{ }^{n} s_{1}{ }^{n} \\
(r=2,3, \cdots, n-1) .
\end{gathered}
$$

If we replace $n$ by $p$ in this formula it results directly that the order of $s_{2} s_{1}$ is $p$ whenever $t_{n-1}$ is identity, that is, whenever the order of the commutative commutator subgroup of the group $G$ generated by $s_{1}, s_{2}$ has less than $p-1$ independent generators. Since the commutator subgroup of $G$ is always of index $p^{2}$ it results that whenever the order of $G$ is less than $p^{p+1}$ it cannot contain any operator of order $p^{2}$, and that $G$ always contains exactly $p+1$ subgroups of index $p$.

When $G$ is of maximal order, two of its subgroups of index $p$ involve no operators of order $p^{2}$, but in the remaining $p-2$ of these subgroups all the operators are of order $p^{2}$ except those found in their common subgroup, which is the commutator subgroup of $G$. Hence each of these groups of maximal order is generated by its operators of order $p$, and whenever $p>2$ each of these groups is also generated by its operators of order $p^{2}$. In the special case when $p=2$, these operators clearly generate only the cyclic subgroup of order 4 . 
The system of groups under consideration is of special interest because it includes $p-2$ non-abelian groups in which every operator except identity is of order $p$, for every possible value of the prime number $p$. The number of these groups for a particular prime number therefore depends upon this prime. The fact that the group generated by $s_{1}, s_{2}$ is completely determined by its order seems also worth noting. UNIVERSITY OF ILUINOIS, December, 1916.

\section{A THEOREM IN THE ANALYSIS OF REAL VARIABLES.}

BY PROFESSOR R. L. BORGER.

(Read before the American Mathematical Society, April 21, 1916.)

In this paper the following theorem is proved:

Theorem: If the two real functions $U(x, y), V(x, y)$ of the real variables $x, y$ satisfy the following conditions at each point of a closed region $R$ :

(a) $U$ and $V$ continuous in $x$ and $y$ jointly;

$$
\frac{\partial U}{\partial x} \equiv U_{1}, \quad \frac{\partial U}{\partial y} \equiv U_{2}, \quad \frac{\partial V}{\partial x} \equiv V_{1}, \quad \frac{\partial V}{\partial y} \equiv V_{2}
$$

exist and are finite;

(c) $\Delta U=h U_{1}+k U_{2}+\rho_{1}(h, k), \quad \Delta V=h V_{1}+k V_{2}+\rho_{2}(h, k)$;

(d) $\operatorname{Lim}_{h, k \doteq 0} \frac{\left|\rho_{i}(h, k)\right|}{|h|+|k|}=0 \quad(i=1,2)$;

(e) $U_{1}=V_{2}, \quad U_{2}=-V_{1}$

then $U$ and $V$ are analytic functions of $x, y$ in $R$.

An immediate consequence of the theorem is that if any function $W$ of a complex variable $z$ possesses a finite derivative at each point of a simply connected closed region $R$ then:

1. This derivative is continuous.

2. All the derivatives of $W$ exist.

3. The function $W$ may be represented by a power series in $z$. 